\title{
Design and Implementation of an On-Demand Home Power Management System based on a Hierarchical Protocol
}

\section{AUTHOR(S):}

Maeda, Tomotaka; Nakano, Hiroki; Morimoto, Naoyuki; Sakai, Kazumi; Okabe, Yasuo

\section{CITATION:}

Maeda, Tomotaka ...[et al]. Design and Implementation of an On-Demand Home Power Management System based on a Hierarchical Protocol. Computer Software and Applications Conference (COMPSAC), 2015 IEEE 39th Annual 2015, 3: 188-193

\section{ISSUE DATE:}

2015

URL:

http://hdl.handle.net/2433/198744

\section{RIGHT:}

(C) 2015 IEEE. Personal use of this material is permitted. Permission from IEEE must be obtained for all other uses, in any current or future media, including reprinting/republishing this material for advertising or promotional purposes, creating new collective works, for resale or redistribution to servers or lists, or reuse of any copyrighted component of this work in other works.; この論文は出版社版でありません。引用の際には出版社版をご確認ご利用ください。;This is not the published version. Please cite only the published version. 


\title{
Design and Implementation of an On-Demand Home Power Management System based on a Hierarchical Protocol
}

\author{
Tomotaka Maeda, Hiroki Nakano, Naoyuki Morimoto, Kazumi Sakai, Yasuo Okabe \\ Kyoto University \\ Kyoto, Japan \\ \{tomo,cas,morimoto\}@net.ist.i.kyoto-u.ac.jp, \{k-sakai,okabe\}@media.kyoto-u.ac.jp
}

\begin{abstract}
We have designed and implemented an on-demand home power management system for saving electric power consumption reliably and systematically without affecting our Quality of Life. The system is based on protocol hierarchy composed of the request/response layer, the path control layer, and the physical layer. Based on the layered protocol architecture, it becomes possible to treat power allocation, power flow setting, and power transmission separately. This makes the on-demand power management system flexibly extensible. In this paper, we describe a use case of our system for power management at a single home, and show how power is saved with least degradation of the Quality of Life in experiments done in a demonstration house.
\end{abstract}

Keywords-HEMS; smart outlet; smart home; energy on demand;

\section{INTRODUCTION}

After Fukushima Daiichi nuclear disaster in March 2011, the demand and the supply of electric power do not balance and power consumption is strictly suppressed, especially in summer and in winter, not only in industries but also at home in Japan.

There have been developed so called HEMS (Home Energy Management Systems) [1] for saving energy at each household. HEMS are based on technologies for remote sensing and automatic control. A HEMS calls ordinary people's attention to energy saving by visualizing power usage in real time, and it also controls air conditioning and lighting automatically to suppress energy consumption based on information from sensors in rooms.

However, there is no enforceable mechanism to stop or restrict power supply to an appliance when it is connected to an outlet. The householders should have responsibility to keep the power consumption within permissible limits.

As a solution of this issue, Matsuyama et al. have proposed Energy on Demand (EoD) mechanism in the concept of i-Energy $[2,3]$. In EoD, an appliance send a message requesting power to a power source, and electric power is supplied after negotiation among the source and appliances considering the power of the source and priority of requests from other appliances. Thus it is possible to keep the power consumed in a house with in a specified limit.

In this paper, we propose an on-demand home power management system based on protocol hierarchy. While existing EoD systems are designed in centralized architecture, our system is in autonomous distributed one based on protocol hierarchy. Power network composed of power routers works in three-layered protocol architecture, the request/response layer, the path control layer, and the physical layer. Treating power allocation, power flow setting, and power transmission in each layer separately, the on-demand power management system is flexibly extensible in attaching or detaching power source equipment and powered devices.

We have designed and implemented a system for saving electric power consumption reliably and systematically without affecting the Quality of Life of ordinary people living in a house. We have investigated a use case of our system for power management at a single home. We show how power is saved with least degradation of the Quality of Life in experiments done in a demonstration house.

The rest of this paper is composed as follows. In Chapter II, the concept of on-demand power supply is described. The threelayer architecture of EoD protocol is shown in Chapter III. In Chapter IV we show the system based on the architecture and in Chapter V we will describe experiments held in a demonstration environment. In Chapter VI concluding remarks are stated and some mention on future works as well.

\section{ON-DEMAND POWER SUPPLY}

In the conventional power network used in our home, electric power generated at power plants comes to home via a switch board of the house and is measured there. Power lines are distributed from the board to outlets on walls, and appliances are connected to power either at outlets or via table taps connected at some outlets. Once an appliance is switched to on, it consumes power which it needs, regardless of the status of other powered devices. If the sum of the power consumed at each appliance excesses the capacity of the switch board, the breaker at the board suddenly works and power supply to all devices is shut down.

In order to avoid this situation, Energy on Demand power network requires each powered device to request its demand of power to the power source explicitly, how much power is needed, before it is powered. Information on demand and supply of electric power is exchanged on the same line where power is conveyed. The flows of energy are managed and visualized with the information.

Based on this concept, Kato et al. [4] proposed a power management system which assures specified power save ratio. Daily integral power consumption is planned beforehand, and 
power is allocated on request from a powered device considering the requested amount of power and the priority of the request which corresponds to the quality of life when the power is supplied (or not).

Our proposed system is designed based on the similar concept, but ours has advantage in granularity of control since it takes not only the amount of instantaneous power but also the path on which the power is supplied in the network from the power source to the powered device in consideration.

\section{THE DESIGN OF OUR EOD SYSTEM}

\section{A. Overview of the System}

Although this is no more than an estimate, a standard EoD network is composed of EoD-aware appliances, EoD-aware power sources, power routers which connect among sources and appliances by wire, and a home gateway.

We assume that there may be more than one power sources in a network. Each appliance sends request for power to all sources, and choose the best offer among replies from them. The system continuously works without interruption when a source or an appliance is attached to or detached from the system, since our system is designed as an autonomous distributed one.

\section{B. The Three-layered Architecture}

We have designed a three-layered hierarchical protocol architecture, composed of the request/response layer, at which EoD-aware devices decides how much power will be received and be sent, the path control layer, at which a path from a source to a powered appliance is established along power routers, and the physical layer, at which electric power is conveyed on wires under physical constraints. Figure 1 shows the hierarchy.

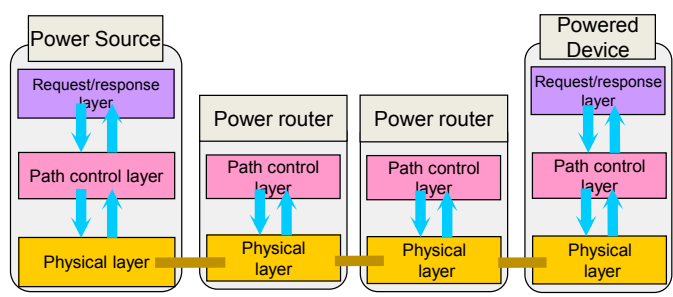

Fig. 1.

The protocol hierarchy

Based on this protocol hierarchy, we can integrate heterogeneous power lines, either AC/DC, Power over Ethernet (PoE), USB, or wireless power supply in a system. In our system, not only the consistency of power demand and supply, but also the constraints on the capacity of wires and devices that convey electricity is considered.

In the protocol hierarchy, the path control layer is the waist of hour-glass model. We may replace the physical layer protocol to another one, and the request/response layer as well.

\section{The request/response layer}

At the request/response layer, each powered device decides how much power it requests, and each power source decides how much it offers in response. Priority of each request is also determined considering the integral power consumption of the day, the situation observed with sensors, and history of past power usage. There is an inevitable trade-off between keeping the QoL of residents within allowable level and suppressing the instantaneous and integral power consumption in the house within permissible level.

\section{The path control layer}

At the path control layer, power sources, powered devices, power routers and the network among them are recognized each other. A path between a power source and a powered device along routers and wires on the network is calculated and established on request.

Even if a power source has enough capacity to supply all the power from devices which request power, there might be less capacity on any path to serve it. In such cases a power path which has already been allocated from the source to some device with less priority might be preempted $[5,6,7]$. The device which has been preempted its power supply will try to get power and a path for power supply by requesting less power for a degraded operation mode.

\section{E. The physical layer}

Actual electric power is transferred among devices and power routers on the physical layer. We assume usage of both AC and DC. There have already been developed DC power routers based on $\mathrm{PoE}[8,9]$ and $\mathrm{AC}$ power routers based on ordinary commercial AC regulation $(100 \mathrm{~V}$ with $50 / 60 \mathrm{~Hz}$ in Japan) [10]. There have also been proposed energy packet networks [11] and DC-packet power routers [12], which packetize electric current.

To adapt ordinary appliances which do not aware EoDprotocols to such intelligent power supply, so called smart taps or smart outlets are utilized $[13,14]$. Smart outlets acts the roles of both power routers and powered devices.

Power transferred by routers or consumed at appliances is continuously measured and is reported to the home gateway periodically.

\section{THE SYSTEM DESIGN}

\section{A. Hardware}

As key components of our system, we have adopted smart outlets that have features for power control [14]. Each of the smart outlets has four outlets, relays for ON/OFF control of outlets each, sensors for instantaneous voltage and current at each outlet, and an embedded computer (Armadillo 440) with an ARM-based CPU with USB and Wi-Fi interfaces. Our system including the EoD protocol stack is developed on the embedded Linux working on it.

\section{B. The EoD protocol}

\section{1) Implementation of the request/response layer}

\section{a) EoD rules}

Our system utilizes a rule-based EoD system by Yoshihisa et al. [15] as the basis of the request/response layer. The system works based on three types of rules, global meta-rules, global rules and local rules. A global meta-rule defines a system-wide policy for reducing power consumption. A global rule is derived from global meta-rules and defines a rule for power request at a specific situation. A local rule defines a rule for power request at a specific operation of each appliance derived from the global 
meta-rules and the specification of the appliance. Each appliance determines power and priority of request.

\section{b) Power request from EoD-aware appliances}

Most of modern home appliances have embedded microcomputers in it for smart operation. Conceptually power request in EoD should be calculated by a microcomputer inherently in each appliance. However, except very few that support ECHONET, most of current smart appliances have no open interface to interact with microcomputers from outside. Instead we utilize the control unit of each appliance as the interface. We have converted the control panel of some appliances like washing machines, microwave ovens and rice cookers so that we can input commands via ZigBee. We have also used IrDA for appliances that have a wireless remote control feature like air conditioner, fan heater, TV sets, video recorders, and cleaners. EoD request and response is processed on the smart outlet connected to the appliance, not on the appliance itself.

Such non-native EoD alliances act in our system as follows. First a person in the home tries to operate an appliance on an EoD-aware remote control unit. Then the signal is received by the smart outlet connected to the appliance. The smart outlet determines the operation based on the local rules and sends request for power required for the action to the power network. If the request is accepted the smart outlet controls the appliance to do the operation. If the power request is not accepted the smart outlet calculates an alternative operation and tries again. If all tries fail the remote control unit inform the person that.

An appliance (or the smart outlet connected to a non-native appliance) periodically resends power request, so that it can get more power as soon as operation of other appliances with higher priority ends. If power which has already allocated is preempted by another appliance, it sends request in the same manner and move to another operation within the allocated power.

Figure 2 shows a sample scenario of EoD at the request/response layer. In this scenario the capacity of power source is $1500 \mathrm{~W}$ and request that exceeds the capacity would be rejected. At the first scene (leftmost) the cleaner works in high power mode $(1100 \mathrm{~W})$ and the air conditioner is in low power mode $(200 \mathrm{~W})$. At the second scene (middle) a person in the room turns the air conditioner into high power mode $(700 \mathrm{~W})$. Here we assume that the priority of request from the air conditioner is higher than that from the cleaner. The sum of power consumption by the two appliances exceeds the limit and the cleaner automatically turns into middle power mode $(650 \mathrm{~W})$. When the air conditioner turns back into lower power mode the cleaner get enough power to operate with higher power mode again.

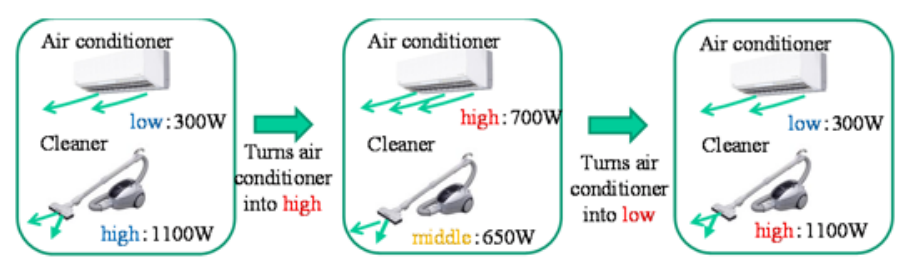

Fig. 2. A sample scenario of Energy on Demand

\section{c) Pseudo EoD by ordinary appliances}

Although we have developed converted appliances controllable via ZigBee and there are appliances which have IrDA remote control interface, there remain many appliances which have no interface for external control. As a simulative implementation, we have developed the following pseudo EoDaware mechanism for such appliances using software on smart outlets.

Assume that an appliance is connected to a smart outlet. When the appliance turns on or changes its operation mode, the smart outlet detects change of power consumption and guesses the current mode. Then the smart outlet sends request for the power usage of the mode to the power network. If the request is accepted the smart outlet just remembers the state. If not it shut downs the power supply to the appliance. We call this post facto EoD.

Since we have no external control to change the operation mode of such appliances, all we can do is just to turn on or off by supplying and stopping the power. Even if an appliance has more than one operation modes, we cannot distinguish them by priority of power request. But besides this restriction, we can deploy ordinary home appliances into our EoD system without any modification of them.

\section{2) Implementation of the path control layer}

We adopt an RSVP-based power allocation protocol as the path control layer protocol by Miyamoto et al. [6] as a reference, and have developed software named EoDresv, which is a simplified implementation of the protocol.

EoDresv uses two types of messages, NodeInfo for ID of each node (appliance, power source or power router) and capacity if it is a source, and LinkInfo for information of each link (upper node ID, lower node ID and link cost) for initial and steady information sharing. These messages are sent from all nodes periodically and they are used in order to grasp the graph topology of the power network, just as link state information is periodically flooded by OSPF-TE in the original protocol [6].

Three types of messages, ReqMsg, PathMsg and ResvMsg, are used for power request, response to request, and allocation of a path. ReqMsg is a message for power request by a powered device and contains a name of the power flow, node ID of the powered device which originates the message and a list for prioritized power requests which the device needs.

PathMsg is a message which shows a response to a request in the list and the path on which power is supplied from a power source and contains name of the power flow, ID of the source, ID of the powered device, the number of requests in the list, the path from the source, and a list of other reservation which will be torn by the request when it is accepted. A PathMsg message is originated by a power source when the source receives a ReqMsg message and some of the requests in the list is acceptable for the source. A PathMsg is transferred along the power routers on the path from the source to the powered device hop by hop, and the information on the path and the list of other reservation to be torn is added.

When a powered device receives a PathMsg message, it sends back along the path on which the PathMsg comes to 
establish a power flow. Figure 3 shows how a power flow between a power source and a powered device is established.

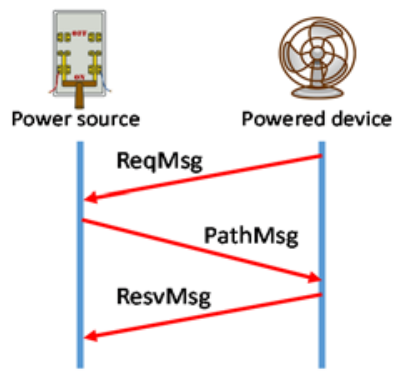

Fig. 3. 3-way hand shake of Energy on Demand

EoDresv supports the situation where there are more than one power sources. In a power network which have multiple power sources, a ReqMsg from a device is received by multiple power sources, and each source independently determines the response to offer and sends back a PathMsg each. When a device receives PathMsg messages from more than one sources for a request, it chooses the best offer among them and reply a ResvMsg message to it. Note that offers from other sources may different in the amount of power, and the cost of the path from the source to the device.

\section{3) Implementation of the physical layer}

Our system supports heterogeneous technologies at the physical layer. As a first step we have deployed ordinary AC $100 \mathrm{~V}, 60 \mathrm{~Hz}$ regulation, compatible with commercial service in west Japan. We use smart outlets described in Section IV-A as power routers. We also utilize AC power routers that have two inputs [16], so that we support a mesh topology, not a tree, where multiple power sources are connected.

The smart outlets have a feature of a software-based circuit breaker, which measures the electric current continuously at each outlet and shut it down when it exceeds the limit for a specified period, like " $120 \%$ excess compared to the allocated value $5 \mathrm{~A}$ continues for more than 15 seconds at outlet \#3." This works for not only post facto EoD but for also unintentional excess of power consumption caused by hardware trouble of devices or wires.

\section{Benefit of the system}

Let us remind what will happen when the power consumption at a home exceeds the limit set at the switch board. Power supplied to the home will suddenly shut down and all the appliances in the house stop. Even after the householder check the cause, remove it and reset the breaker, some operation of appliance like rice cooking does not necessarily be resumed without impacts.

In contrast, only a smallest number of appliances that have lower priority will be stopped (or, with less impact, be changed into a lower power mode) when the power consumption exceeds the limit in our system. This greatly improves our quality of life at such situation.

\section{EXPERIMENTS AND CONSIDERATION}

\section{A. Demonstrative Experiments}

We have deployed the EoD system based on the threelayered architecture described in the previous chapter at Kyo Eco House, a demonstration smart house built by Kyoto Prefecture.

The system composed of rule engine software for post facto EoD and a home gateway at the request/response layer, EoDresv at the path control layer, and home appliances and smart outlets at the physical layer.

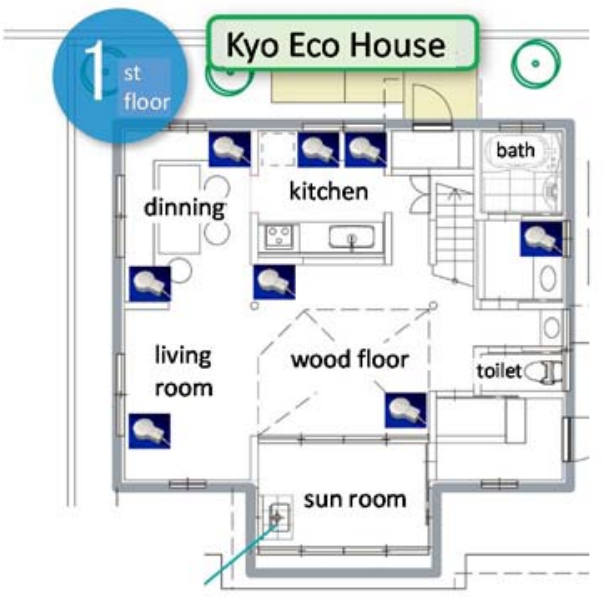

Fig. 4. Sketch of Kyo Eco House

As is shown in Figure 4, we have deployed 8 smart outlets at the first floor of the house, and have used appliances like a washing machine, a coffee maker, a humidifier, a rice cooker, etc. Among them we have done some experiments using a water pot, a rice cooker and a microwave oven based on some scenarios described below.

Figure 5 shows diagrams of power consumption at the three appliances each. The vertical axis shows power consumption (W) and the horizontal axis shows the time clock (second).

Assume that the limit of the breaker on the circuit is $1500 \mathrm{~W}$. If we used these three appliances on the same circuit at a time, the sum would exceed the limit and the breaker should shut off the circuit.

We will show how post facto EoD works in our system, in the following three scenarios in each of which appliances have different priority in power request.

Scenario 1: A rice cooker has already been working and a microwave sends power request with the same priority.

Scenario 2: A water pot has already been working and a microwave sends power request with higher priority

Scenario 3: A microwave has already been working and a water pot sends power request with lower priority.

In each scenario the power consumption exceeds the limit when more than one appliances are turned on at a time. 
Figure 6 shows how much power is consumed in each experiment of Scenario 1-3. The vertical axis shows power consumption (W) and the horizontal axis shows the time clock (second) from the start of the experiment. Green lines show the limit and purple lines shows sum of the power actual consumption at each experiment.

In the experiments, it is observed that preemption occurs only when power request which comes later have higher priority than that of power consumption which has already reserved.

\section{B. Consideration}

In each experiment there is a short term the total power consumption (purple line) exceeds the allowable power limit, $1500 \mathrm{~W}$ (green line). This inevitable if the system works in post fact EoD. It takes around 3 seconds to shut down the power supply to the appliance of lower priority. In the experiments, the EoDresv collects data from all smart outlets once a second. It would be possible to collect data more frequently but it would increase the communication overhead among the home gateway and the devices.

It remains intrinsic restriction in post fact EoD that power request can be sent based only on a single operation mode. Even

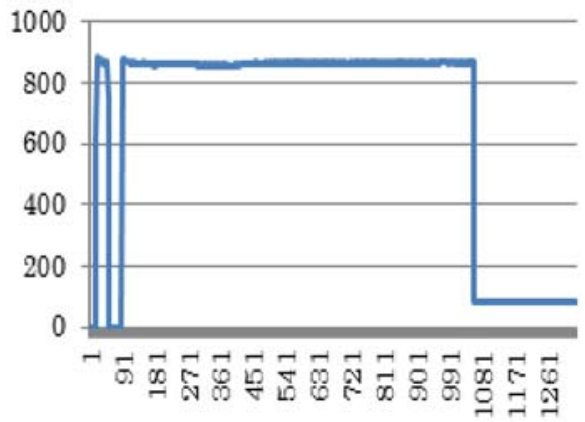

(a) Water pot

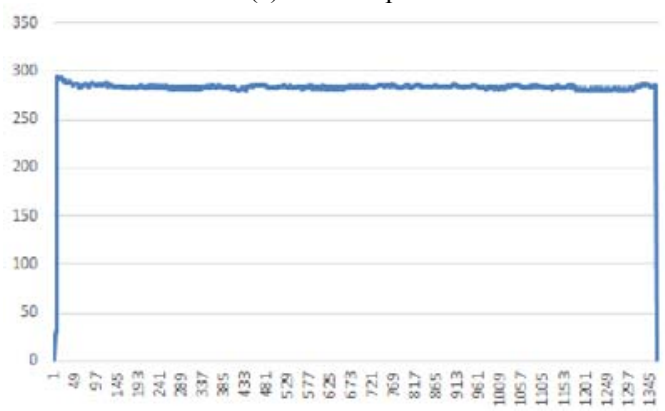

(b) Rice cooker

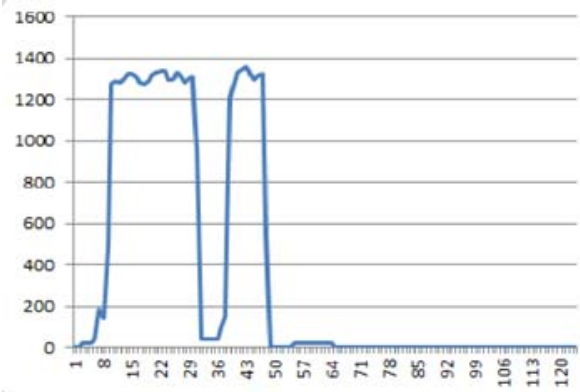

(c) Microwave oven

Fig. 5. Power consumption at each appliance if the appliance has more than one operation modes, it cannot fall back to another mode when it fails to get power in the mode requested. We really hope that smart appliances which have interfaces to be controlled will become popular in near future.

\section{CONCLUDING REMARKS}

We have designed and implemented an on-demand home power management system for saving electric power consumption reliably and systematically based on a threelayered hierarchical protocol, the request/response layer, the path control layer, and the physical layer. We have shown how power is saved in experiments done in Kyo Eco house, a demonstration house in Kyoto.

We have shown some results of experiments based on simple scenarios. More extensive investigation using more appliances is future work.

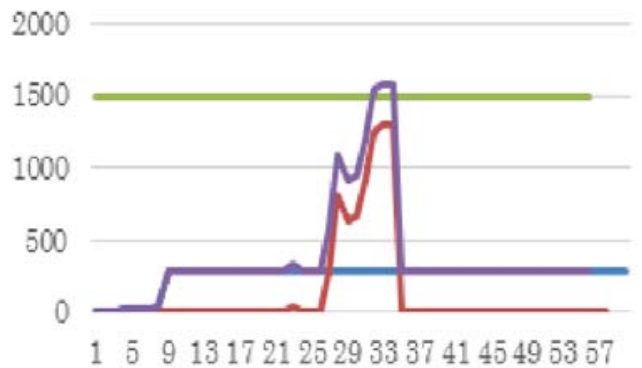

(a) Scenario 1

(Blue line: rice cooker, red line: microwave oven)

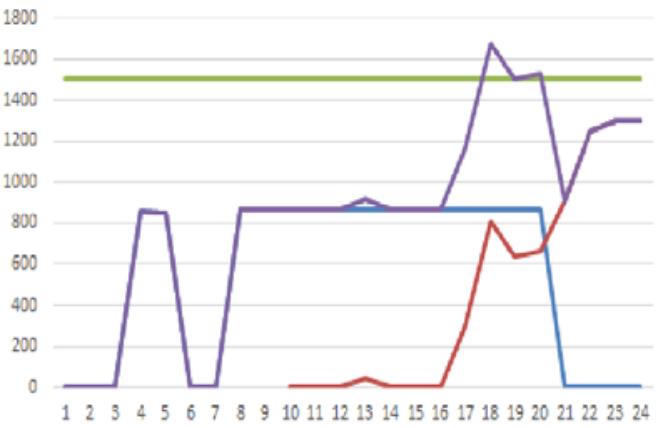

(b) Scenario 2

(Blue line: water pot, red line: microwave oven)

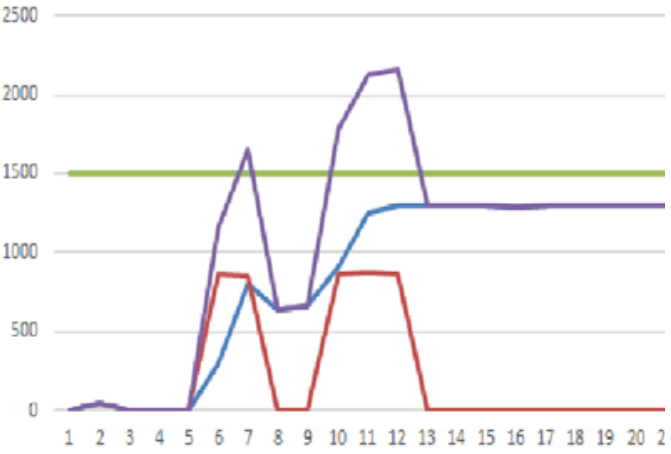

(c) Scenario 3

(Blue line: microwave oven, red line: water pot)

Fig. 6 Power consumption in post fact EoD 


\section{ACKNOWLEDGMENT}

The authors would express our sincere appreciation to Mr. Okamoto and Mr. Koyama at TNT, Dr. Hoshino at Octopath, and Mr. Murakami at Daiwa House Industry, for their support and suggestions in design of the system and the experiments during this research. This work was supported by NICT Advanced Telecommunication Research Fund and JST Super Cluster Program.

\section{REFERENCES}

[1] M Inoue, T. Higuma, Y. Ito, N. Kushiro, H. Kubota, "Network architecture for home energy management system," IEEE Trans. Consumer Electronics, vol. 49, pp.606-613, Aug. 2003

[2] T. Matsuyama, "Creating safe, secure, and environment-friendly lifestyles through i-Energy, New Breeze, vol.21, no. 2, pp.1-8, Apr. 2009

[3] T. Matsuyama, "i-Energy: smart demand-side energy management," chapter 7, Smart Grid Applications and Developments, Springer, 2014 pp. 141-163.

[4] T. Kato, K. Yuasa, T. Matsuyama, "Energy on demand:Efficient and versatile energy control system for home energy management," 2011 IEEE International Conference on Smart Grid Communications, pp.392397, Oct. 2011.

[5] K. Sakai, Y. Okabe, Quality-aware Energy Routing toward On-demand Home Energy Networking, Proc. IEEE Consumer Communications and Networking Conference (CCNC) 2011 (Special Session on Ecological Home Network), pp.1041-1044, Jan. 2011.

[6] T. Miyamoto, Y. Koyama, K. Sakai, Y. Okabe, "A GMPLS-based power resource reservation system toward Energy-on-Demand home networking," 12th IEEE/IPSJ International Symposium on Applications and the Internet (SAINT2012), pp.138-147, July 2012.

[7] M. Yokohata, T. Maeda, Y. Okabe, "Power allocation algorithms of PoE for on-Demand power supply," 1st IEEE International Workshop on Consumer Devices and Systems (CDS2013), (Proc. IEEE COMPSAC2013 Workshops), pp.517-522, July 2013.
[8] T. Shibata, K. Sakai, Y. Okabe, "The design and implementation of an on-demand DC grid in home," 11 th IEEE/IPSJ International Symposium on Application and the Internet (SAINT2011), pp.152-159, Jul. 2011.

[9] M. Yokohata, T. Maeda, Y.Okabe, "An extension of the Link Layer Discovery Protocol for on-demand power supply network by PoE," First International WorkShop on Energy-Aware Systems, Communications and Security (EASyCoSe 2013) (In conjunction with IEEE AINA-2013), pp. 1612-1616, March 2013.

[10] T. Takuno, Y. Kitamori, R. Takahashi, T. Hikihara, "AC power routing system in home based on demand and supply utilizing distributed power sources," Energies, vol 4, pp. 717-726, May 2011.

[11] E. Gelenbe, "Energy packet networks: ICT based energy allocation and storage," in Lecture Notes of the Institute for Computer Sciences, Social Informatics and Telecommunications Engineering; GreeNets, Rodrigues, J.J.P.C., Zhou, L., Chen, M., Kailas, A., Eds.; Springer, vol. 51, pp. 186195, 2011.

[12] R. Takahashi, T. Takuno, T. Hikihara, "Estimation of power packet transfer properties on indoor power line channel," Energies, vol. 5, pp.2141-2149, June 2012.

[13] T. Kato, HS Cho, D. Lee, T. Toyomura, T. Yamazaki, “Appliance recognition from electric current signals for information-energy integrated network," 7th International Conference on Smart Homes and Health Telematics (ICOST): Ambient Assistive Health and Wellness Management in the Heart of the City, pp. 150-157, July 2009.

[14] N. Morimoto, M. Tanaka, T. Akehi, M. Yoshida, H. Yoshimizu, M. Takiyamada, Y. Kamimura, "The design and implementation of a smart tap for policy-based power management," Proc. IEEE Consumer Communications and Networking Conference (CCNC) 2012 (Special Session on Ecological and Smart Home Network), pp.296-300, Jan. 2012.

[15] T. Yoshihisa, N. Fujita, M. Tsukamoto, "A rule-based home energy management system for power consumption reduction," INFORMATION Journal, vol.16, no.9(B), pp.6893-6904, Sept. 2013.

[16] N. Morimoto, Y. Fujita, M. Yoshida, H. Yoshimizu, M. Takiyamada, T. Akehi, M. Tanaka, "Policy-based power router with power sensors for efficient allocation of energy," 1st IEEE Global Conference on Consumer Electronics (GCCE 2012), pp.477-480, Oct. 2012. 